the resulting 2-cell be amalgamated with $\alpha_{2}$ across $c_{2}$ to obtain a 2 -cell $\rho$ with $p$ for boundary. By Theorem 2.1, $\rho$ is the interior of $p$. Since $\alpha_{1}$ and $\alpha_{2}$ are exterior to $c$, the 2-cell $\alpha$ constitutes the entire interior of $c$. The Jordan-Schoenflies Theorem now follows readily in all its generality.

UNIVERSITY OF ILLINOIS

\title{
A NOTE ON CURVATURE AND BETTI NUMBERS
}

\section{H. GUGGENHEIMER}

1. S. Bochner has proved the following theorem $[2]:{ }^{1}$ Let $M^{(m)}$ be a closed manifold with complex structure $[4 ; 7]$ of complex dimension $m$, on which there exists a Kähler-metric $[2 ; 3 ; 5]^{2}$

$$
\begin{aligned}
d s^{2} & =g_{i k^{*}}\left(d z^{i} d z^{*}\right),^{3} \\
\frac{\partial g_{i k^{*}}}{\partial z_{l}} & =\frac{\partial g_{l k^{*}}}{\partial z_{i}} .
\end{aligned}
$$

Let $R_{i k^{*}}$ denote the Ricci tensor and

$$
P_{h i^{*} j k^{*}}=R_{h i^{*} j k^{*}}-\frac{1}{m+1}\left(g_{h i^{*}} R_{j k^{*}}+g_{h k^{*}} R_{i^{*} j}\right)
$$

the tensor of projective curvature. In every point of $M^{(m)}$ we form the numbers

$$
\begin{aligned}
& L=\inf _{\xi} \frac{-R_{i k^{*}} \xi^{i \xi k^{*}}}{\xi^{i} \xi_{i}}, \\
& P=\sup _{\xi}\left|\frac{P_{h i^{*} j k^{*} \xi^{h i^{*}} \xi^{j k^{*}}}}{\xi^{h i^{*}} \xi_{h i^{*}}}\right|,
\end{aligned}
$$

with all vectors $\xi^{i}$ and skew-symmetric tensors $\xi^{i j^{*}}$ attached to the point in question. If

$$
L>0
$$

Received by the editors December 8, 1950.

${ }^{1}$ Numbers in brackets refer to the bibliography at the end of the paper.

2 Products of differentials in parentheses denote ordinary products, products without parentheses are skew products.

${ }^{3}$ We denote by $i^{*}$ the index relative to $z^{* i}$. 
holds everywhere on $M^{(m)}$, and if, for some $p$, we have at all points the relation

$$
(p-1) P<\left(1-\frac{p-1}{m+1}\right) L,
$$

then for the Betti numbers $p^{q}$ of $M^{(m)}$ we have

$$
\begin{array}{rlrl}
p^{2 k} & =1, & 2 k & \leqq p, \\
p^{2 k+1} & =0, & 2 k+1 \leqq p .
\end{array}
$$

2. The proof of this theorem in [2] is based on Bochner's lemma [1]:

If on a compact (differentiable) manifold we have, for a given scalar $\Psi, \Delta \Psi \geqq 0$ everywhere, then $\Delta \Psi=0$ everywhere.

By the theory of harmonic integrals $[5, \S 2]$ the group of complex harmonic differential forms of degree $q$ on $M^{(m)}$ is isomorphic to the $q$ th cohomology group with real coefficients of $M^{(m)}$. The form

$$
\Omega=g_{i k} d z^{i} d z^{* k}
$$

with all its powers $\Omega^{k}, k \leqq m$, is harmonic and not equal to $0[3 ; 5 ; 6]$. It is known that every harmonic differential form $\phi^{q}$ of degree $q \leqq m$ is a sum with constant coefficients $c_{i}$,

$$
\phi^{q}=\sum_{i=0}^{[q / 2]} c_{i} \chi^{q-2 i} \Omega^{i},
$$

where the forms $\chi$ are harmonic and "effective," that is, satisfy the condition

$$
* \Omega * \chi=0 .
$$

A differential form $\phi_{(h)}^{q}$ is said to be pure, and of type $h$, if it is a homogeneous form of degree $h$ in the differentials $d z^{* i}$. Every harmonic form is a sum of pure harmonic forms. Let $p_{(h)}^{q}$ be the rank of the linear space of the pure harmonic forms on $M^{(m)}$ of degree $q$ and type $h$. Then

$$
\begin{aligned}
p^{2 k} & \equiv p_{(k)}^{2 k}(\bmod 2), \\
p^{2 k+1} & \equiv 0(\bmod 2) .
\end{aligned}
$$

(14) has been proved first by Lefschetz in the case of algebraic manifolds.

If $\phi^{q}$ is a pure form $\phi_{(h)}^{q}$, then (11) becomes [5] 


$$
\stackrel{q}{\phi(h)}=\sum c_{i} \chi_{(h-i)}^{q-2 i} \Omega^{i} .
$$

3. We now prove the following theorem.

THEOREM. If on $M^{(m)}$ we have (6) and (7) everywhere, then there exist no effective harmonic forms of type $k$ and degree $2 k$ for $k \leqq p$, that is, we have

$$
p^{2 k} \equiv 1(\bmod 2), \quad 2 k \leqq 2 p .
$$

From (8), (9), (14), and (15) we see that we have the following situation if (6) and (7) hold everywhere on $M^{(m)}$ :

$$
\begin{array}{rlrlrl}
p^{2 k} & =1 & \text { for } & 2 k \leqq p, & p^{2 k} \equiv 1(\bmod 2) & \text { for } 2 k \leqq 2 p, \\
p^{2 k+1}=0 & \text { for } & 2 k+1 \leqq p, & p^{2 k} \equiv 0(\bmod 2) & \text { always. }
\end{array}
$$

Proof. It is a fundamental property of a Kähler metric [5] that only those components of the curvature tensor which are equal to one of the form $R_{h j^{*} k l^{*}}$ are not equal to 0 . Let $\psi_{(k)}^{q}=P_{i_{1}} \cdots i_{q-k_{q}} i_{q-k+1} \cdots i_{q}^{*}$ $d z^{i_{1}} \cdots d z^{* i_{q}}$ be an effective form of degree $q$ and type $k$. Then (12) is

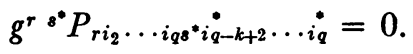

Let $\Psi\left(\psi_{(k)}^{q}\right)$ be the scalar $P_{i_{1}} \ldots i_{q}^{*} P^{i_{1}} \cdots i_{q}^{*}$ and $k \leqq q-k$. Condition (16) gives $[2,(36)]$

$$
\begin{aligned}
& \frac{1}{2} \Delta \Psi=P_{i_{1} \ldots i_{q}, \lambda} P^{i_{1}} \cdots i_{q}^{*}, \lambda
\end{aligned}
$$

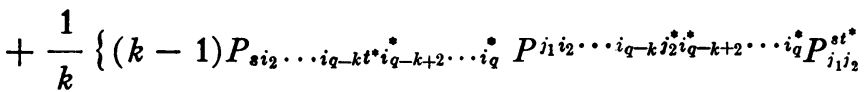

$$
\begin{aligned}
& -\left(1-\frac{k-1}{m+1}\right) P_{i_{1} \ldots s^{*}} \ldots i_{q}^{*} P^{i_{1}} \cdots t^{*} \ldots i_{q}^{*} R_{t^{*}}^{8^{*}} .
\end{aligned}
$$

This formula shows that from (6) and (7) follows

$$
\Delta \Psi\left(\psi_{(k)}^{2 k}\right)>0,
$$

a contradiction to Bochner's lemma.

4. The theory of effective harmonic forms and (11) is not restricted to Kähler manifolds [5]. In fact, if on a Riemannian manifold $M^{(m)}$ of dimension $2 m$ a 2 -form exists,

$$
\Omega=h_{i k} d x^{i} d x^{k},
$$

which is closed and is everywhere of the same rank $2 \rho$, then (11) is 
valid for $q \leqq \rho$, the effective forms being defined analogously to (12) as forms $\phi^{q}=F_{i_{1}} \ldots i_{q} d x^{i_{1}} \cdots d x^{i_{q}}$ satisfying

$$
h^{i k} F_{i k i_{8}} \ldots i_{q}=0 \text {. }
$$

Let $P_{h i j k}$ be defined as

$$
P_{h i j k}=R_{h i j k}-\frac{1}{m+1}\left(g_{h i} R_{j k}+h_{h k} R_{i j}\right) .
$$

It is easy to see that (6) and (7) imply (8) and (9) for $2 k$ resp. $2 k+1$ $\leqq \min (p, \rho)$.

\section{BiBLIOGRAPHY}

1. S. Bochner, Curvature and Betti numbers, Ann. of Math vol. 49 (1948) pp. 378390.

2. - Curvature and Betti numbers II, Ann. of Math. vol. 50 (1949) pp. 77-93.

3. B. Eckmann and H. Guggenheimer, Sur les varietes closes a metrique hermitienne sans torsion, C.R. Acad. Sci. Paris vol. 229 (1949) pp. 503-505.

4. Ch. Ehresmann. Sur la théorie des espaces fibrés. Colloque de Topologie Algébrique, Paris, 1949, pp. 3-15.

5. H. Guggenheimer, Ueber komplex-analytische Mannigfaltigkeiten mit Kahlerscher Metrik, Comment. Math. Helv. vol. 25 (1951) no. 4.

6. W. V. D. Hodge, The theory and applications of harmonic integrals, Cambridge, 1941.

7. H. Hopf, Zur Topologie der komplexen Mannigfaltigkeiten, Studies and Essays presented to R. Courant, New York, 1948, pp. 167-185.

BAsel, SwTtZerLaNd 